\title{
Spontaneous Inflammatory Pelvic Disease in Adult Non-Castrated Female Rats Treated With Estrogen
}

\author{
Aristóteles M G Ramos², Sandro Perazzio ${ }^{1}$, \\ Aroldo F. de Camargos ${ }^{2}$ and Fausto E.L. Pereira ${ }^{1}$
}

\begin{abstract}
Biomedical Centre of the Federal University of Espírito Santo ${ }^{1}$, Vitória, ES; Department of Ginecology and Obstetrics of Medicine School of Federal University of Minas Gerais², Belo Horizonte, MG, Brazil
\end{abstract}

\begin{abstract}
The adaptive immune response of the genital tract is under the control of sexual steroids; however, the influence of sex hormones on innate immune mechanisms of the genital mucosa are only beginning to be understood. We found that long-term estrogen treatment increases the risk for inflammatory pelvic diseases in adult non-castrated female rats. Female rats $(110 \mathrm{~g}$ to $130 \mathrm{~g})$ received estrogen (10 rats; $17-\beta$ estradiol, 50 mg pellet; 10 rats: subcutaneous weekly injection of estradiol valerate $0.166 \mathrm{mg} / \mathrm{kg}$ ). Ten rats received a pellet of $17-\beta$ estradiol and were treated with amoxicillin, $50 \mathrm{mg} / \mathrm{kg}$ after the $90^{\text {th }}$ day of exposure to estrogen. Three control groups of ten rats were also used. The estrogen-treated rats developed an inflammatory pelvic disease, with abscess formation after the third month of hormonal treatment. All the surviving animals were killed after six months of hormonal exposure. Among 15 survivors of the two groups that received estrogen 13 animals presented tuboovarian abscesses. Among eight survivors of the group treated with amoxicillin, six had tuboovarian abscesses. None of the 30 control rats presented macro or microscopic signs of inflammatory disease in the uterus, tubes or ovaries. We conclude that estrogen impairs the defense mechanisms of the genital tract of non-castrated female rats, enhancing bacterial growth in the vagina and ascending infection to the uterus, tubes and ovaries. Key Words: Inflammatory pelvic disease, estrogen, mucosal immunity, rats.
\end{abstract}

The mucosal immune system in the female genital tract is the first defense against pathogenic microorganisms [1,2]. While it is known that adaptive immune responses in the genital mucosa are under hormonal regulation, with separate and distinct mechanisms in the vagina and uterus $[3,4]$, the hormonal control of innate immune mechanisms in the genital tract is only beginning to be explored [1]. Estrogens decrease antigen presentation and secretion of immunoglobulin in the vaginal mucosa of female rats [5-7] and increase the vaginal growth of Candida albicans [8,9]. In contrast, estrogens increase the Received on 05 September 2004; revised 11 January 2004. Address for correspondence: Dr. Fausto E.L. Pereira. Núcleo de Doenças Infecciosas Centro Biomédico UFES. Av. Marechal Campos 1468 Maruípe. Zip code: 29040-091 Vitória ES Brazil.

The Brazilian Journal of Infectious Diseases 2005;9(1):6-8 (C) 2005 by The Brazilian Journal of Infectious Diseases and Contexto Publishing. All rights reserved. immune response in the endometrium, and they increase resistance against Chlamydia inoculated in the vagina $[4,10]$. On the other hand, estrogens increased the susceptibility to Chlamydia infection in female guinea pigs $[11,12]$.

As the effect of long-term estrogen exposition in non castrated female rats on the resistance of the genital mucosa to infection had not been investigated, we developed a study to examine this system.

Young female adult Wistar rats weighing $110 \mathrm{~g}$ to $130 \mathrm{~g}$, maintained in plastic cages with 6 to 10 animals, receiving a commercial diet and tap water "ad libitum", were used in three experiments. In experiment 1,10 rats received estrogen by subcutaneous implant of a $50 \mathrm{mg}$ pellet of 17-beta-estradiol (Akzo-Nobel, Netherlands) subcutaneously in the midline dorsum, and 10 rats were maintained as controls. In experiment 2,10 rats received estrogen by weekly subcutaneous injection of estradiol valerate $(0.166 \mathrm{mg} / \mathrm{kg}$; Smart 
Figure 1. Uteri of female rats after six months treatment with estrogen. A: Bilateral tubo-ovarian abscess. B: Pyometra in the two horns of uterus. C: Opened uterine abscess: there is some purulent material in the cavity. D: Uterus without inflammatory disease, showing dilatation of the uterine horns. E: Animal without abscess: endometritis with leukocytes in the stroma (arrow head) and in the lumen of endometrial glands (arrow); H\&E, 400x. F: Uterus with pyometra: purulent exudate (pe) in the lumen and inflammatory exudate in the uterine wall (UW); H\&E, 100X).

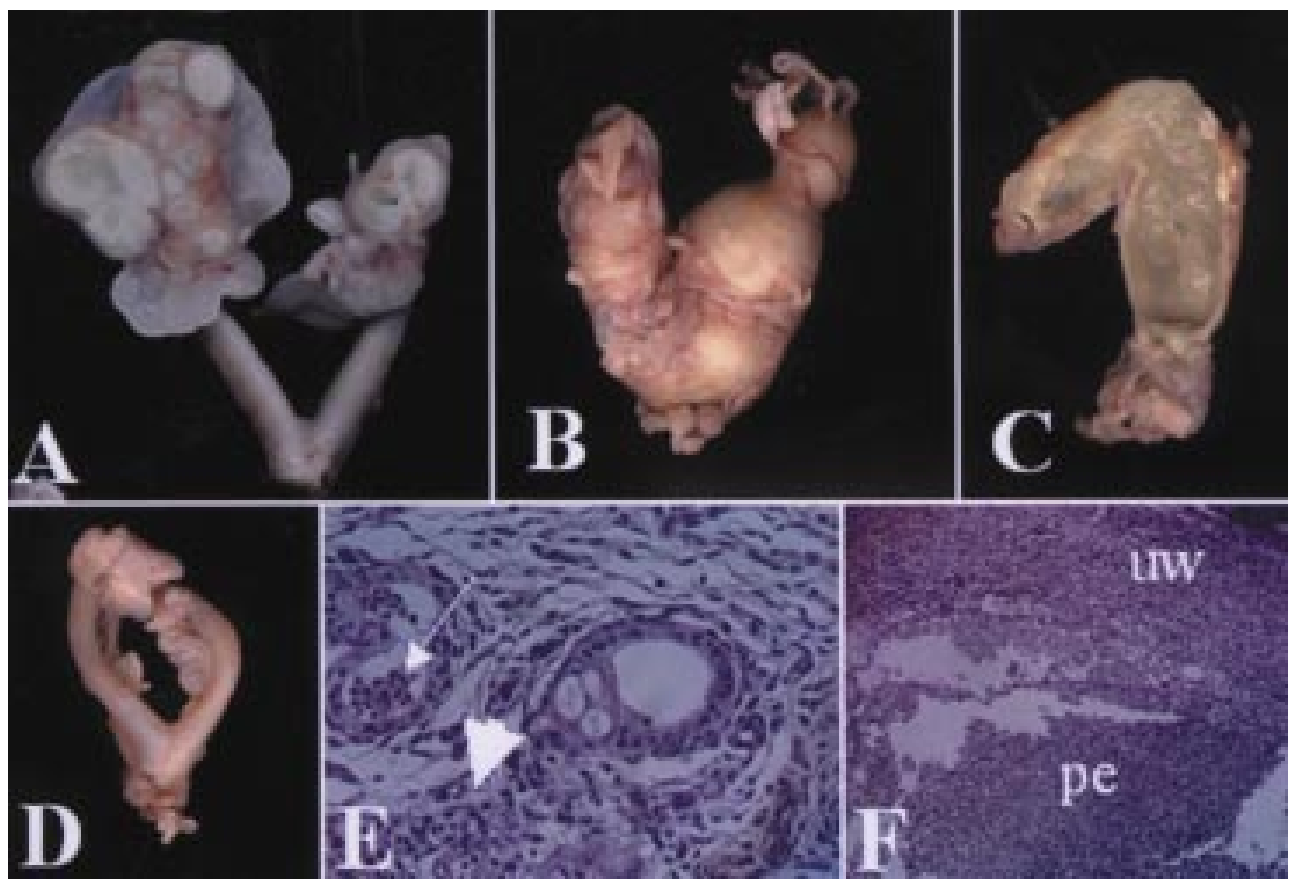

Chemische Produkte Handels, Germany) in corn oil, and 10 control rats received weekly injections of corn oil. In experiment 3, 10 rats received a pellet of 17 beta-estradiol and were treated with amoxicillin (50 $\mathrm{mg} / \mathrm{kg}$ ), daily, after 90 days of hormonal treatment, and 10 rats were used as controls, without estrogen or amoxicillin treatment. All the surviving animals were killed with pentobarbital anesthesia, six months after beginning hormonal treatment.

All the rats treated with estrogen presented weight loss, which was significant after 90 days of hormonal exposure. Three and four rats, from experiments 1 and 2 , respectively, died spontaneously between 90 and 120 days. Autopsies were performed on three animals and all presented inflammatory pelvic disease with abscess formation in the ovary, tubes and uterus. The surviving rats were killed during the next 30 days, and six and four rats, respectively, from experiments 1 and 2 , presented tuboovarian abscess and pyometra
(Figure 1:A, B and C.). The three animals without inflammatory pelvic disease had a dilated uterus with a cloudy fluid in the lumen. In experiment 3 , in which the animals received amoxillin, two animals died after between 120 and 180 days of hormone exposure. Among the eight survivors, six had tuboovarian abscesses and pyometra as observed in experiments 1 and 2, and two had a dilated uterus, without signs of inflammatory disease (Figure 1D).

The microscopic study of the vagina of the rats with and without evident abscesses showed hyperplasia of the epithelium, with hyperkeratinization and large numbers of eosinophils in the stroma. There was hyperplasia and keratinization of the epithelium near the vagina in the uterine cervix (corresponding to the ectocervix). Focal areas of epidermoid metaplasia and eosinophils were found in the stroma of the endometrium. There were leukocytes in the stroma and in the lumen of the endometrial glands of some animals 
(Figure 1E). In abscessed uteri, the histological picture was that of pyogenic inflammation: the abscess wall presented massive exudation of neutrophils and macrophages, with destruction of normal structures and extensive peripheral fibrosis (Figure 1F). The Gramstained smears of pyogenic material showed gram positive and Gram-negative bacteria. We did not culture this material.

In the control groups $(n=30)$, none of the animals showed macro or microscopic evidence of inflammation in the reproductive tract and ovaries.

We conclude that long-term estrogen administration in non-castrated female rats increases the risk for inflammatory pelvic disease. Several mechanisms could be involved in this increased risk for ascending infection induced by estrogen: (a) a reduction in immune adaptive mechanisms in the vaginal mucosa has been reported after short-term estrogen exposure in castrated rats [36]; (b) impaired production of soluble microbiocidal factors by epithelial cells of the genital mucosa (secretory leukocyte protease inhibitor, defensins, lysozime, lactoferrin) has been demonstrated in the genital tract [13-15]; (c) keratinization of the vaginal and cervical epithelium could facilitate the adhesion of pathogens and impair other innate defense mechanisms, enhancing the ascending invasion of microorganisms. Studies are in progress in our laboratory to establish the etiology of this inflammatory pelvic disease. In addition, we are developing an experimental model of ascending inflammatory pelvic disease in rats treated with estrogen and inoculated in the vagina with fecal bacteria.

\section{References}

1. Wira C.R., Fahey J.V. The innate immune system: gatekeeper to the female reproductive tract. Immunology 2004;111(1):13-5.

2. Brandtzaeg P. Mucosal immunity in the female genital tract. J Reprod Immunol 1997;36(1-2):23-50.

3. Wira C.R., Sandoe C.P. Effect of uterine immunization and oestradiol on specific IgA and IgG antibodies in uterine, vaginal and salivary secretions. Immunology 1989;68(1):24-30.
4. Wira C.R., Kaushic C. Mucosal immunity in the female reproductive tract: effect of sex hormones on immune recognition and responses. In: H. Kiyono, P.L. Ogra, J.R. MacGhee [eds.] Mucosal vaccines: new trends in immunization. Academic Press, New York, NY 1996; pp. 375-386.

5. Wira C.R., Rossoll R.M., Kaushic C. Antigen-presenting cells in the female reproductive tract: influence of estradiol on antigen presentation by vaginal cells. Endocrinology 2000;141(8):2877-85.

6. Wira C.R., Roche M.A., Rossoll R.M. Antigen presentation by vaginal cells: role of TGFbeta as a mediator of estradiol inhibition of antigen presentation. Endocrinology 2002;143(8):2872-9.

7. Wira C.R., Rossoll R.M. Oestradiol regulation of antigen presentation by uterine stromal cells: role of transforming growth factor-beta production by epithelial cells in mediating antigen-presenting cell function. Immunology 2003;109(3):398-406.

8. Sobel J.D., Muller G., McCormick J.F. Experimental chronic vaginal candidosis in rats. Sabouraudia 1985;23(3):199206.

9. Fidel P.L. Jr., Cutright J., Steele C. Effects of reproductive hormones on experimental vaginal candidiasis. Infect Immun 2000;68(2):651-7.

10. Kaushic C., Zhou F., Murdin A.D., Wira C.R. Effects of estradiol and progesterone on susceptibility and early immune responses to Chlamydia trachomatis infection in the female reproductive tract. Infect Immun 2000;68(7):4207-16

11. Rank R.G., White H.J., Hough A.J. Jr., et al. Effect of estradiol on chlamydial genital infection of female guinea pigs. Infect Immun 1982;38(2):699-705.

12. Barron A.L., Pasley J.N., Rank R.G., et al. Chlamydial salpingitis in female guinea pigs receiving oral contraceptives. Sex Transm Dis. $1988 ; 15(3): 169-73$.

13. Moriyama A., Shimoya K., Ogata I., et al. Secretory leukocyte protease inhibitor (SLPI) concentrations in cervical mucus of women with normal menstrual cycle. Mol Hum Reprod 1999;5(7):656-61.

14. Valore E.V., Park C.H., Quayle A.J., et al. Human betadefensin-1: an antimicrobial peptide of urogenital tissues. J Clin Invest 1998; 101(8):1633-42.

15. Valore E.V., Park C.H., Igreti S.L., Ganz T. Antimicrobial components of vaginal fluid.Am J Obstet Gynecol 2002;187(3):561-8. 